\title{
Clinical assessment of the effectiveness of treatment for hand-foot skin reaction, caused by the targeted antitumor therapy with multikinase inhibitors of angiogenesis
}

\author{
E.A. Shatokhina ${ }^{1,2}$, L.S. Kruglova ${ }^{1}$, O.A. Shukhov ${ }^{2}$ \\ ${ }^{1}$ Central State Medical Academy, Moscow, Russia \\ ${ }^{2}$ National Medical Research Center for Haematology, Moscow, Russia
}

Introduction. Effective treatment of the hand-foot skin reaction (HFSR) is an essential component of the comprehensive treatment of patients receiving antitumor therapy with multikinase angiogenesis inhibitors.

Methods. 29 patients were under study, they received antitumor treatment with multikinase angiogenesis inhibitors (sorafenib, lenvatinib) and displayed clinical signs of II-III degree HFSR.

Results. The 15 patients of the experimental group (EG) received a combination of alpha-lipoic acid, 600 $\mathrm{mg}$ per day, and an ointment containing $0.005 \%$ calcipotriol and $0.05 \%$ betamethasone dipropionate twice a day. The 14 patients of the control group (CG) received treatment with a combination of dermatic medications - a cream containing 10\% urea and an ointment containing $0.05 \%$ betamethasone dipropionate. After a week, the regression of skin symptoms was more prominent within EG including erythema, paresthesia, pain, and burning sensation; the Dermatology Life Quality Index (DLQI) has shown greater improvement compared to $C G$ with the statistical significance of $p<0.05$. After 2 weeks the DLQI was 17,4 within $E G$ and 22,8 within $C G(p<0.0001)$. After 3 weeks $E G$ has demonstrated greater improvement in erythema, skin desquamation, paresthesia $(p<0.0008)$, pain $(p<0.0001)$, and DLQI $(p<0.0001)$. At the end point of the study (after 4 weeks) the improvements in symptoms and DLQI were greater in EG compared to CG with the statistical significance of $p<0.0001$, correlation presented in table .

Table. Correlation between Dermatology Life Quality Index and clinical signs of HFSR

\begin{tabular}{|r|r|r|r|r|r|r|r|}
\hline & erythema & pain & $\begin{array}{c}\text { burning } \\
\text { sensation }\end{array}$ & paresthesia & $\begin{array}{c}\text { skin } \\
\text { desquamation }\end{array}$ & fissures & blisters \\
\hline $\begin{array}{r}\text { Dermatology Life Quality } \\
\text { Index (DLQI, 0-30) } \\
\text { (Spearman rank } \\
\text { correlation) }\end{array}$ & 0,8741 & 0,8576 & 0,8392 & 0,8258 & & & \\
\hline $\mathrm{p}$ & $<0,0001$ & $<0,0001$ & $<0,0001$ & $<0,0001$ & & & \\
& & & & & & \\
\end{tabular}

Conclusions. Patients receiving targeted therapy with multikinase inhibitors are recommended to receive treatment for HSFR with a combination of alpha-lipoic acid per os and topical therapy with an ointment containing calcipotriol and betamethasone dipropionate. 